\title{
Management of Post-LASIK Keratoectasia with a Piggyback Contact Lens System
}

\author{
Lacey Haines, BSc, OD \\ Luigina Sorbara, OD, MSc, FAAO, \\ FBCLA
}

University of Waterloo 200 University Avenue, West Waterloo, ON

N2L 3G1

\begin{abstract}
This case report highlights the clinical challenges involved in managing a patient with post-LASIK keratoectasia. A single case is presented with a focus on contact lens fitting and concurrent ocular surface disease. A review of the literature indicates that current screening methods prior to refractive surgery have made improvements for detecting patients at higher risk of serious complications. Possible treatment options for iatrogenic keratoconus include contact lenses, intrastromal corneal ring segments, corneal cross linking, and keratoplasty. This report demonstrates that post-LASIK keratoectasia with concurrent ocular surface disease can be successfully managed with a piggyback contact lens system
\end{abstract}

KEY WORDS:

Post-LASIK keratoectasia, ectasia, piggyback lenses, post-LASIK dry eye, gas permeable contact lenses

\section{Résumé}

L'article décrit les défis cliniques soulevés par la prise en charge d'un patient atteint d'une kératectasie post-LASIK. Un seul cas est présenté; on met l'accent sur l'ajustement de la lentille et une affection concomitante de la surface oculaire. Selon la littérature, les méthodes de dépistage actuelles permettent de mieux identifier, avant une chirurgie réfractive, les patients qui risquent de présenter des complications graves. Plusieurs traitements existent pour le kératocône iatrogène, dont les lentilles de contact, les anneaux cornéens intrastromaux, la réticulation de la cornée et la kératoplastie. Le cas rapporté ici montre que l'on peut traiter efficacement une kératectasie avec affection concomitante de la surface oculaire grâce à un système de lentilles de contact piggyback.

\section{MOTS CLÉS:}

Neuropathie optique héréditaire de Lever, réadaptation de la basse vision, multidisciplinaire, habilitation compétitive, counselling 


\section{INTRODUCTION}

Post-LASIK keratoectasia is a rare but significant vision-threatening complication of refractive surgery. ${ }^{1}$ Because this condition is seldom seen, the actual incidence is unknown, ${ }^{2,3}$ but some estimates have ranged from $0.04 \%^{4}$ to $0.2 \%{ }^{5}$ The onset of the condition is variable, occurring days to years after the refractive surgery procedure. ${ }^{1,2,6}$ The signs and symptoms of iatrogenic keratoectasia resemble keratoconus with progressive corneal thinning and steepening, increasing myopia and irregular astigmatism, and loss of best-corrected visual acuity (BCVA).,2,7 Two distinct clinical presentations are identified in the literature, one being described as a central ectasia with little irregular astigmatism and good BCVA that occurs in normal eyes and the other described as a paracentral ectasia more similar to keratoconus with irregular astigmatism and poor BCVA that more often occurs in eyes that have undetected preoperative keratoconus or forme fruste keratoconus. ${ }^{8-10}$ Recent histopathological studies of post-LASIK ectasia have implied that it shares a common histopathology with keratoconus, with the exception of lack of endothelial changes seen in the iatrogenic form. ${ }^{11}$

Prevention through pre-screening and identifying poor candidates is essential for avoiding this serious complication. ${ }^{9}$ Although there are some established risk factors identified in the literature, they do not explain all cases of post-LASIK ectasia. Abnormalities in preoperative topography are regarded as the most significant risk factor; others include younger age, reduced and/or asymmetric preoperative pachymetry, lower residual stromal bed thickness (RSBT), higher refractive correction, steep preoperative corneal curvature, correction for against-therule or oblique astigmatism, number of enhancement procedures, eye rubbing, family history of keratoconus, refractive instability, and male sex. , $2,4,6,7,9,12,13^{-13}$

Treatment alternatives usually begin with non-surgical options; patients with mild forms of iatrogenic ectasia can benefit from spectacles or traditional soft contact lenses. However, more advanced cases require specialty contact lenses, such as rigid gas-permeable (GP), scleral, hybrid, or piggyback contact lenses (PBCL)., ${ }^{1,7,14}$ With a corneal shape that is more oblate and irregular, contact lens fitting tends to be more difficult in post-refractive-surgery eyes compared to their non-surgical counterparts. Evidence shows that more chair time is required, there is an increased frequency of office visits, an increased number of lenses are usually required to complete the fit, and more post-refractive-surgery eyes fail contact lens wear. ${ }^{15,16}$

If contact lenses are not successful, there are several surgical alternatives. Intrastromal corneal ring segments have been shown to increase unaided visual acuity, as well as decrease refractive cylinder and spherical equivalent refractive error., ${ }^{2,17}$ Corneal cross linking has the ability to increase the biomechanical strength of the compromised cornea, allowing stabilization of progressive post-LASIK ectasia, as well as improve corrected visual acuity. ${ }^{2,7}$ Keratoplasty may also be offered as a treatment for iatrogenic ectasia, with the literature favouring anterior lamellar keratoplasty over penetrating keratoplasty due to the reduced complications., ${ }^{2,12,18}$

Piggyback contact lenses involve a soft contact lens (SCL) worn therapeutically under a GP lens, allowing increased comfort with a SCL while maintaining the optical advantages provided by a GP lens. ${ }^{10,19}$ Positive-powered lenses are most commonly used for the SCLs because the steeper anterior surface aids in centering the GP lens. ${ }^{20-22}$ However, negative powers can be useful for individuals who would like to wear SCLs alone, ${ }^{20}$ and one study reported decreased anterior optical aberrations in patients using minus-powered lenses in piggyback contact lens systems. ${ }^{21}$ Advantages of the PBCL system include better centration and stability of the GP lens, increased comfort, improved patient tolerance, and corneal protection-all of which promote improvements in visual acuity. ${ }^{19-22}$

Disadvantages involve concerns about oxygen availability; however, recent evidence suggests that corneal physiological requirements are met as long as both the SCL and GP lenses are made in high oxygen permeable materials and adequate movement of the lenses is achieved. ${ }^{19,20,22}$ Doubling the number of lenses to handle and maintain decreases convenience, but this is somewhat alleviated with daily disposable replacement schedules for the soft piggyback lens. 
Ocular surface disease following refractive surgery correction is a relatively frequent complication. As many as $95 \%$ of patients have symptomatic dryness immediately following LASIK, the percentage of which decreases over time..$^{23}$ Although estimates of incidence vary considerably, there is general agreement that only a small percentage of individuals develop chronic and severe dry eyes. ${ }^{1,2,24,25}$ The primary mechanism is believed to be corneal denervation from flap creation and surface ablation, which results in reduced corneal sensitivity, resulting in decreased feedback to the lacrimal gland through the reflex pathway in the cornea-lacrimal gland functional unit. Consequently, there are decreased neurotrophic influences on epithelial cells, decreased basal and reflex tearing, and decreased blink rate, leading to decreased tear clearance and increased tear evaporation. Abnormal nerve regeneration in the years following LASIK may play a role in persistent ocular surface disease.,24-28 Other factors contributing to ocular surface disease after refractive surgery include alterations in corneal shape that disrupt tear film distribution, as evidenced by iron deposition lines on the cornea, ${ }^{2,25-28}$ and possible damage to limbal goblet cells by the use of a suction ring. ${ }^{25-28}$ Risk factors for developing post-LASIK dry eye in the literature include pre-existing dry eye disease, Asian descent, female sex, lid margin disease, higher refractive correction, deeper ablation depth, and thicker flap..$^{1,2,27}$ Preoperative screening is imperative for reducing the frequency of this common complication, including a thorough case history and preoperative testing. ${ }^{1-28}$ Treatment of existing ocular surface abnormalities and lid disease will also help prevent the onset of this complication. ${ }^{1,25,26,28}$ Other factors, such as the use of femtosecond lasers, nasally hinged flaps, and wider flap hinges, have inconclusive evidence to support their role in preventing ocular surface disease after LASIK. ${ }^{25-28}$

Treatments for post-LASIK ocular surface disease are similar to those for non-surgical eyes, such as artificial tears, identification and treatment of lid disease, punctal occlusion, cyclosporine A, short- term corticosteroid use, autologous serum, bandage soft contact lens, scleral contact lenses, temporary tarsorrhaphy, and amniotic membrane transplant. ${ }^{1,2,25-28}$ Future treatment options might also include topical nerve growth factor., ${ }^{1,25-28}$ Corneal ectasia and dry eye syndrome are challenging post-LASIK complications to treat in isolation, and their coexistence can compound problems, making management even more difficult.

\section{CASE REPORT}

L.P. was a 34-year-old man who had uneventful LASIK performed in 1999 at age 23 years. There were no concerns with his general health and no family history of keratoconus. Although the clinic where the LASIK was performed has since closed, our clinic was able to obtain preoperative corneal topography maps and information from follow-up appointments up to three months after the procedure. His corneal topographies before the surgery are shown in Figure 1 and his pre-LASIK data are summarized in Table 1. Interestingly, the ablation depths were greater than average for the intended refractive correction, but after reviewing the surgeon's notes and the literature, we were unable to learn why this occurred. The topography readings in Figure 1 show abnormal topographies with some steepening of these anterior and posterior corneal surfaces on the elevation maps, more so in the left eye. 


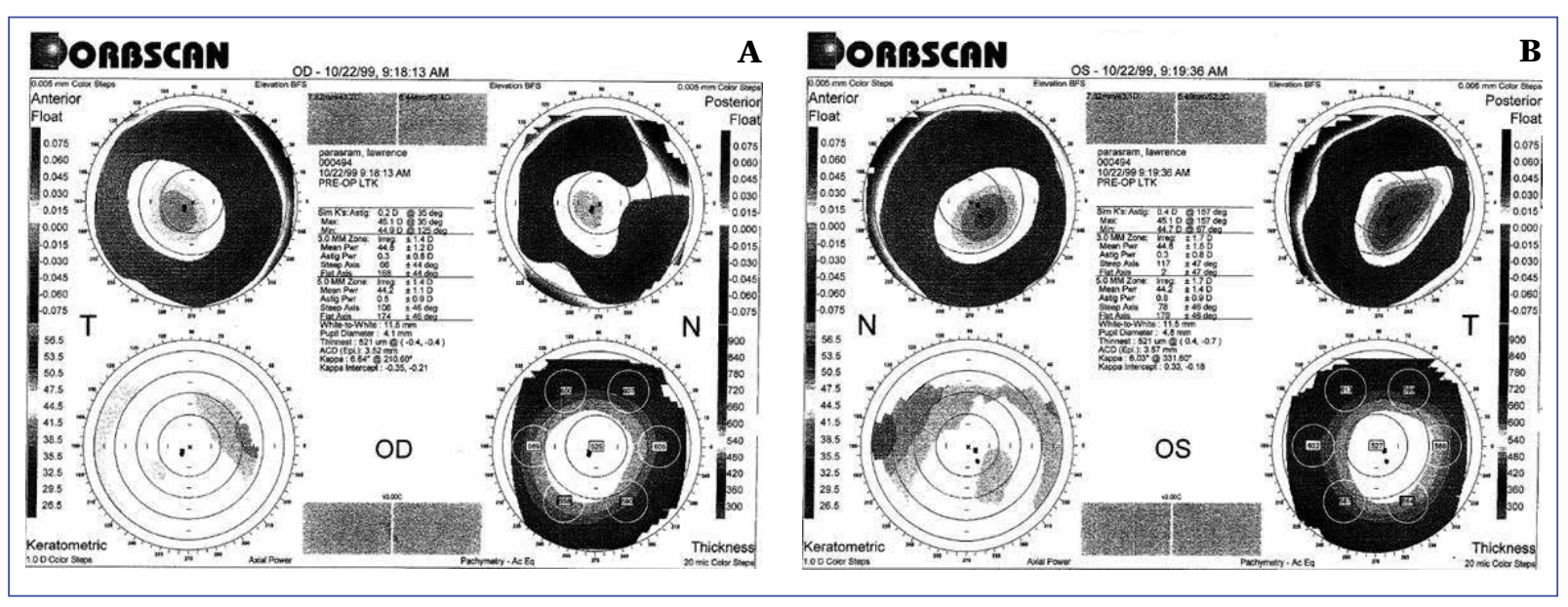

Figure 1. Patient's pre-LASIK corneal topographies in the (A) right and (B) left eyes. Steepening on the anterior and posterior elevation maps can be seen in both eyes.

Table 1. Relevant Ocular Data for Patient Prior to the LASIK Procedure in 1999

\begin{tabular}{|l|l|l|c|c|}
\hline Eye & Manifest Rx & Visual Acuity & Pachymetry $(\boldsymbol{\mu m})$ & Simulated K readings \\
\hline OD & $-3.25-0.25 \times 180$ & $20 / 20-3$ & 521 & $44.9 @ 125 ; 45.1 @ 035$ \\
\hline OS & $-3.75-0.50 \times 075$ & $20 / 20-3$ & 522 & $44.7 @ 067 ; 45.1 @ 157$ \\
\hline
\end{tabular}

Table 2. Information for Patient's LASIK Procedure with a Superior Flap Hinge in Both Eyes

\begin{tabular}{|l|l|l|c|c|c|c|}
\hline Eye & $\begin{array}{l}\text { Attempted } \\
\text { Correction }\end{array}$ & $\begin{array}{l}\text { Zone Diameter } \\
(\mathbf{m m})\end{array}$ & $\begin{array}{c}\text { RSBT* } \\
(\boldsymbol{\mu m})\end{array}$ & $\begin{array}{c}\text { Ablation } \\
(\boldsymbol{\mu m})\end{array}$ & $\begin{array}{c}\text { Header } \\
(\boldsymbol{\mu m})\end{array}$ & Laser Used \\
\hline OD & $-3.50-0.25 \times 180$ & 6.1 & 272 & 69 & 180 & Keracor 217 \\
\hline OS & $-4.00-0.50 \times 075$ & 6.1 & 259 & 83 & 180 & Keracor 217 \\
\hline
\end{tabular}

${ }^{*}$ RSBT $=$ Residual Stromal Bed Thickness

Information about the LASIK procedure is given in Table 2. The flap hinge was located superiorly.

At one-week follow-up, micro-striae were noted in the right eye and mild epithelial ingrowth was seen inferior temporally in the left eye. At three-month follow-up, there were no micro-striae noted and the epithelial ingrowth in the left eye was considered stable at $<0.5 \mathrm{~mm}$ in from the edge of the flap. The manifest refraction at this time was OD $+0.50-0.50$ $\times 160,20 / 20(6 / 6)$ and OS $+0.75-0.50 \times 040,20 / 20(6 / 6)$. Corneal topography in the right eye showed $0.2 \mathrm{D}$ (axis $123^{\circ}$ ) of corneal astigmatism with corneal radii of flat $41.9 \mathrm{D}$ and steep 42.1 D. The left eye showed $0.7 \mathrm{D}$ (axis $28^{\circ}$ ) of corneal astigmatism with corneal radii of flat 40.7D and steep 41.4D.

We had no further ocular history information for L.P., until an ophthalmologist referred him to our clinic in 2009 (10 years later) for contact lens management of irregular corneas. At this time, L.P. had been experiencing decreasing vision over the previous year, with the left eye worse than the right. His unaided visual acuity was 20/60 (6/18) in the right eye and 20/400 (6/120) in the left eye. Corneal topography showed central corneal thinning and steepening, with the left eye more advanced than the right. The manifest refraction was OD $-0.75-1.50 \times 025,20 / 40+2$ $(6 / 12+2)$ and OS +0.25 -1.75 × 120, 20/400 (6/120) in the left eye. He was fit with Rose K2 IC and Rose K (Blanchard Laboratories, Montreal, Canada) corneal GP lenses in the right and left eyes, respectively, and was able to achieve visual acuities of 20/25 (6/7.5) in each eye. L.P. remained in these lenses for the next three years, during which there was some progression of the central steepening that was worse in the left eye, and the left lens was modified to reflect these changes. 
During the same time period, L.P. presented to our clinic on several occasions with ocular irritation and superficial punctate keratitis (SPK) that was treated with topical dexamethasone $0.1 \%$ and tobramycin $0.3 \%$ ophthalmic drops. It was also recommended that he use artificial tears (SYSTANE ${ }^{\circledR}$ Ultra Lubricant Eye Drops, Alcon Laboratories, Inc., Fort Worth, US) for the dryness and discomfort. He was referred to an ophthalmologist for a consultation regarding corneal cross-linking surgery, where it was determined he was not an appropriate candidate.

In 2012, progression was noted again in both eyes and the patient's lenses were becoming increasingly uncomfortable, particularly in the left eye. The manifest refraction was OD -4.00 $-0.75 \times 020,20 / 30(6 / 9)$ and OS: $-7.25-0.50 \times 008,20 / 30-2(6 / 9-2)$. Both GP lenses showed a flat-fitting relationship centrally, and lenses with steeper base curves were ordered for both eyes. Before delivering the new lenses, the patient returned to the clinic with ocular irritation and SPK in both eyes, but worse in the right. The patient was told to discontinue lens wear temporarily and was prescribed tobramycin again. Once this episode resolved and the lenses were delivered, the right lens continued to show excessive central bearing, so the right eye was refit with a Rose K Post Graft (Blanchard Laboratories) corneal GP lens. At the next follow-up visit, the patient reported persistent and decreasing comfort, especially in the right eye, and his corneas continued to show significant SPK in both eyes, now worse in the left eye. The left Rose $\mathrm{K}$ GP lens was modified to a steeper base curve and the patient was instructed to continue with artificial tears.

At his next visit, L.P.'s dryness and discomfort symptoms seemed to have improved in the left eye but persisted in the right eye. He was also developing issues with lens deposits that appeared worse on the right lens. For treatment of the SPK and to increase comfort, the patient was instructed to continue with SYSTANE ${ }^{\circledR}$ Ultra Lubricant Eye Drops (Alcon Laboratories, Inc.) four times a day, and Liposic ${ }^{\circledast}$ Ophthalmic Drops (Bausch + Lomb, Rochester, US) at night were recommended for added therapeutic effect. The patient was also instructed to use Boston ${ }^{\circledast}$ One Step Liquid Enzymatic Cleaner (Bausch + Lomb) in addition to his usual cleaning regimen with Boston Advance ${ }^{\circledast}$ Cleaner and Conditioning Solution (Bausch + Lomb) for the lens deposits. Furthermore, we recommended a lid hygiene routine of hot compresses and lid scrubs to treat a mild underlying meibomian gland dysfunction.

At the next follow-up visit, there were improvements regarding the lens deposits, but the patient continued to experience increased dryness symptoms, and his ocular surfaces showed significant dryness and SPK. The patient reported to be using the artificial tear eye drops about 10 times per day; the pattern of SPK revealed 3 o'clock and 9 o'clock staining from corneal surface desiccation. The artificial tear therapy was switched to Preservative Free SYSTANE ${ }^{\circledR}$ Ultra Lubricant Eye Drops as needed during the day and SYSTANE ${ }^{\circledR}$ Gel Drops at night.

At a subsequent visit, L.P. reported no improvement in his dryness symptoms, and he was becoming increasingly intolerant of his GP lenses. Both lenses showed some inferior displacement, acceptable axial edge clearance, and a three-point touch fitting pattern with fluorescein; however, the left lens showed more than the ideal amount of central bearing (Figure 2). At this time, L.P.'s visual acuities were 20/25+1 (6/7.5+1) in the right eye and 20/252 (6/7.5-2) in the left eye. Slit lamp examination revealed marked ocular surface disease and significant SPK at the 3 o'clock and 9 o'clock regions of both eyes. A particularly severe area of coalesced dehydration staining was noted nasally in the right eye (Figure 3). It was decided that a piggyback contact lens (PBCL) system would be implemented, and the patient was fit with 1-DAY ACUVUE ${ }^{\circledast}$ TruEye $^{\circledast}$ (J\&J, Jacksonville, FL) silicone hydrogel daily disposable contact lenses in both eyes as a bandage lens under his current GP lenses. Although the GP lenses showed improved centration with good movement of both the soft and rigid lenses and acceptable fluorescein patterns in both eyes (Figure 4), there was still relatively greater central bearing of the GP lens in the left eye. Visual acuities with the PBCL system were 20/25+2 (6/7.5+2) in the right eye and 20/25-1 (6/7.5-1) in the left. The final lens parameters for the GP and soft contact lenses are given in Table 3. L.P. was instructed to continue the lid hygiene regimen, Preservative Free SYSTANE ${ }^{\circledR}$ Ultra, Boston ${ }^{\circledR}$ cleaning system and to rinse his GP lenses thoroughly with saline prior to insertion to ensure the SCLs are not contaminated with the GP solution. 

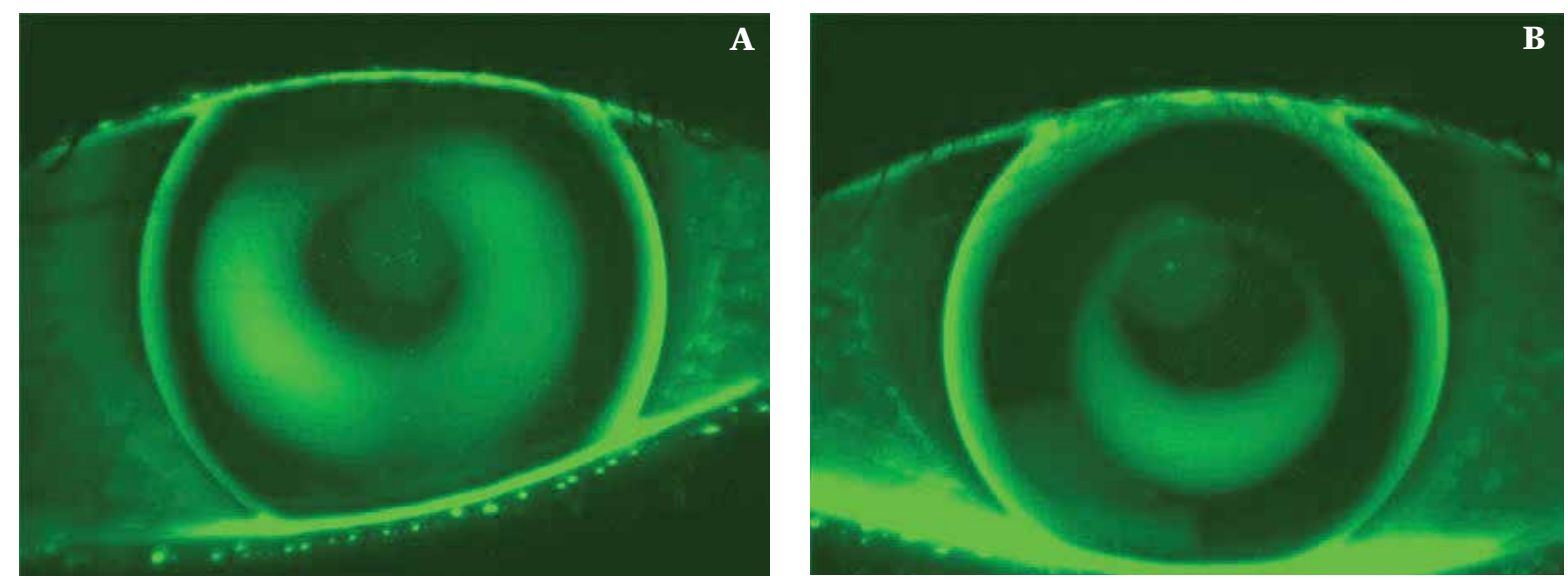

Figure 2. Fit of GP lenses for patient prior to use of PBCL system. (A) Patient's right eye fit with a Rose $K$ Post Graft corneal GP lens. (B) Patient's left eye fit with a Rose K corneal GP lens.

Figure Footnote: $G P$ = gas permeable; $P B C L=$ piggyback contact lens.

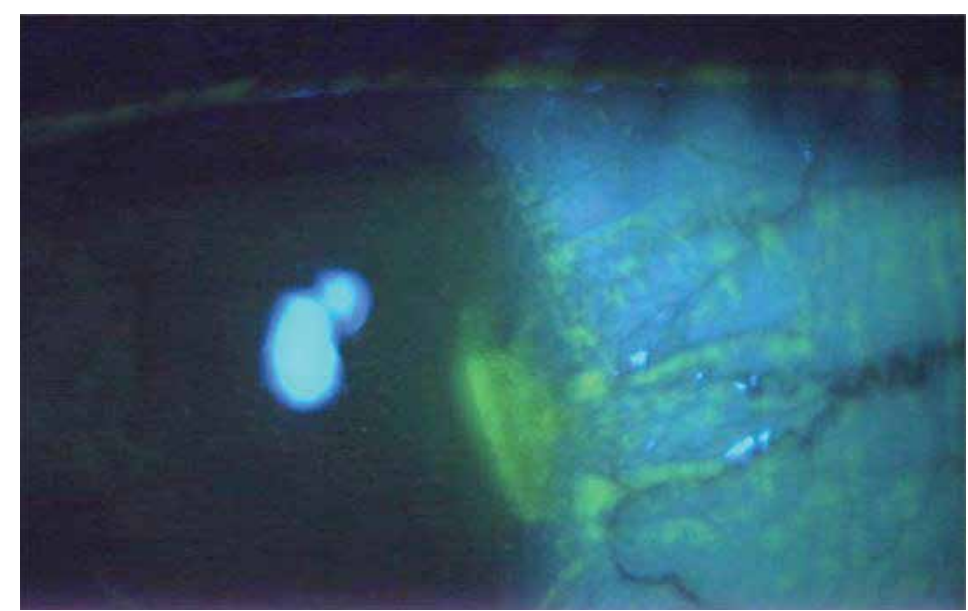

Figure 3. Severe coalesced SPK from dehydration in the nasal region of patient's right eye prior to implementation of the PBCL system.

Figure Footnote: $S P K=$ superficial punctate keratitis; $P B C L=$ piggyback contact lens.
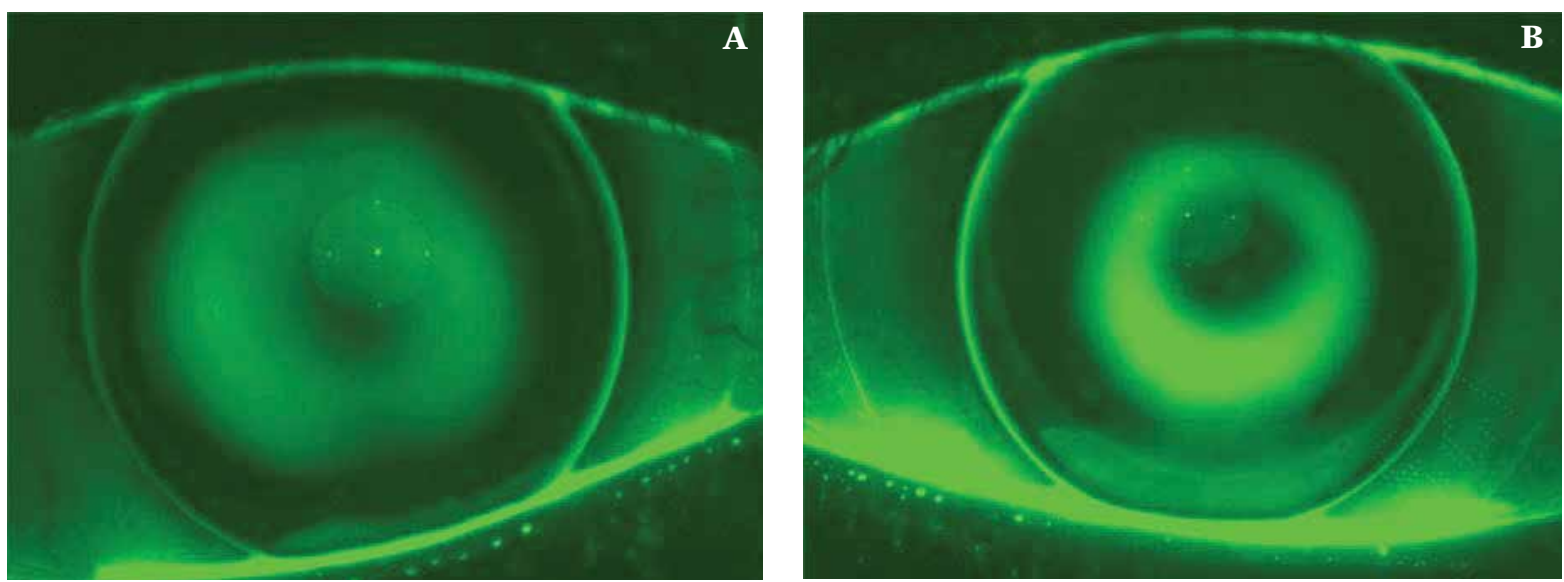

Figure 4. $P B C L$ fit for patient's $(A)$ right and $(B)$ left eyes shows improved centration and decreased central bearing. 
Table 3. Final Lens Parameters for Patient's Soft- and Gas-Permeable Lenses Used in the PBCL System

\begin{tabular}{|l|l|l|c|c|c|c|}
\hline Eye & Lens Name & Material & $\begin{array}{c}\text { BOZR } \\
(\mathbf{m m})\end{array}$ & $\begin{array}{c}\text { Diameter } \\
(\mathbf{m m})\end{array}$ & Power (D) & $\begin{array}{c}\text { Dk } \\
(\times \mathbf{1 0}-11)\end{array}$ \\
\hline OD & 1-DAY ACUVUE TruEye & Narafilcon A & 8.5 & 14.2 & -0.50 & 100 \\
\hline OS & 1-DAY ACUVUE TruEye & Narafilcon A & 8.5 & 14.2 & -0.50 & 100 \\
\hline OD & Rose K Post Graft & Boston XO & 7.5 & 10.2 & -4.00 & 100 \\
\hline OS & Rose K & Boston XO & 6.6 & 9.6 & -8.62 & 100 \\
\hline
\end{tabular}

At one-week follow-up, L.P. reported improved comfort and decreased symptoms of dryness. In fact, he reported his need for artificial tears had reduced to only a couple of times a day. Visual acuities with the PBCL system were 20/20-3 (6/6-3) in the right eye and 20/25+2 (6/7.5+2) in the left. The GP lenses again showed acceptable centration and fluorescein patterns, and there was good movement of both lenses. His ocular surfaces showed dramatic improvements with only trace corneal staining in the right (Figure 5) and left eyes. Because the patient's signs and symptoms were significantly reduced, it was decided there was no further need to address the increased central bearing of the GP lens in the left eye. The patient was instructed to continue with the same PBCL system and cleaning regimen and to use the artificial tears as needed.

\section{CO-MANAGEMENT: YOUR PATIENTS COME FIRST!}

Our Co-Management program offers many advantages:

- Over 30 clinics across Canada

- LASIK MD clinics annually perform over $60 \%$ of all laser vision correction procedures in Canada

- Free laser vision correction for optometrists and optometry students
- More than 10 years experience

- Experienced surgeons who have collectively performed over 750,000 procedures

- Affordable prices for your patients starting at $\$ 490$ per eye*

Direct access to our team: comanagement@lasikmd.com

Book your free consultation at 1-877-793-1515 or at lasikmd.com

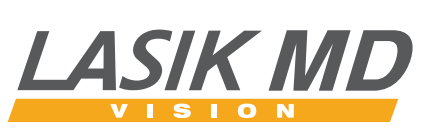

\section{Special rates}

for opticians, optometric assistants and optometry clinic employees.

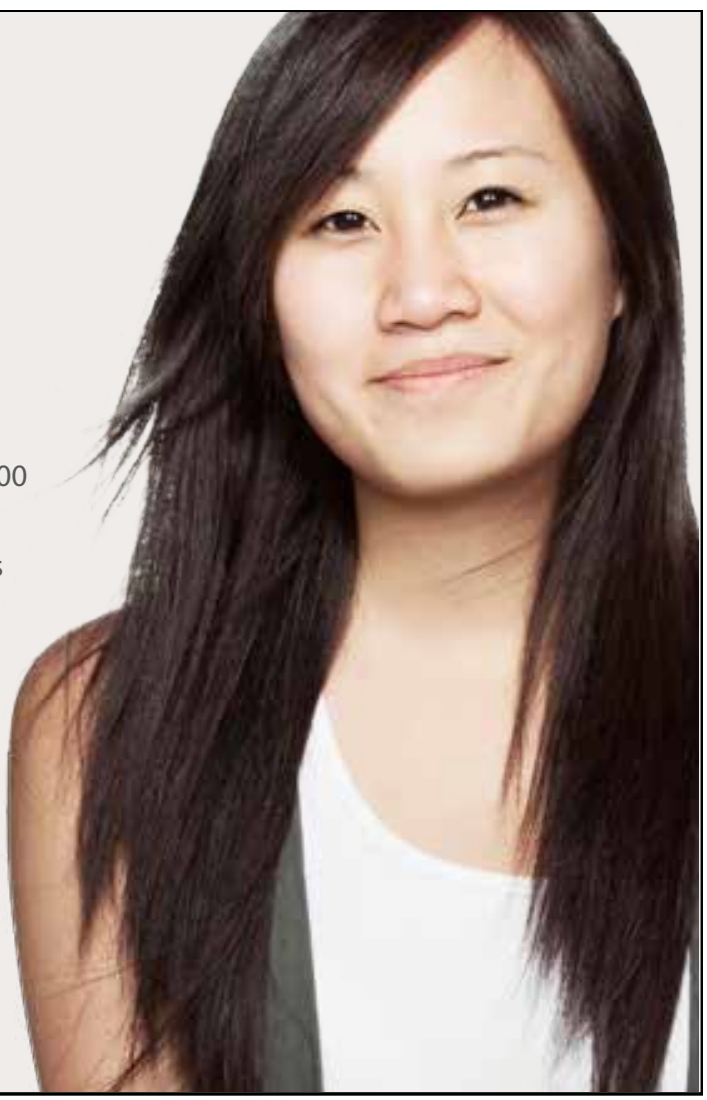

*Prices are subject to change without prior notice and vary based on prescription strength. Only applicable on a procedure for both eyes. Other conditions may apply. 


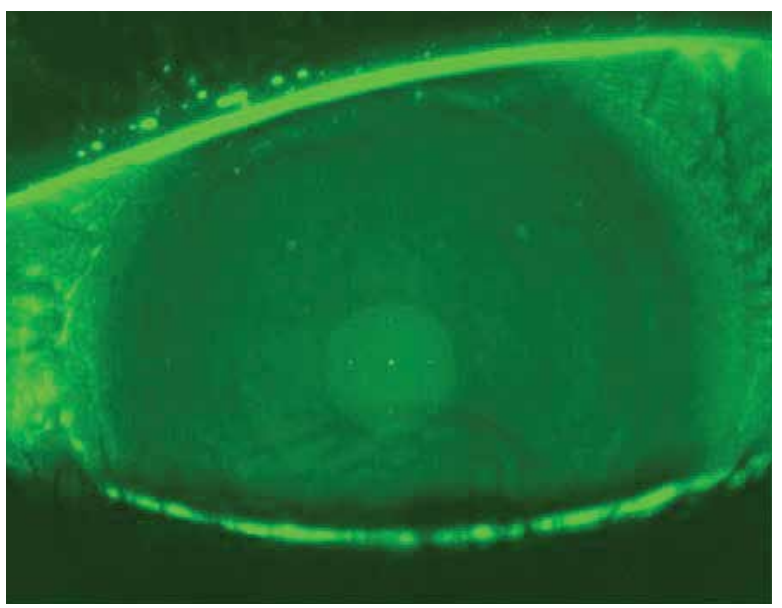

Figure 5. Patient's right eye after implementing the PBCL system showing significant improvement in the condition of his ocular surface.

Figure Footnote: $P B C L=$ piggyback contact lens.
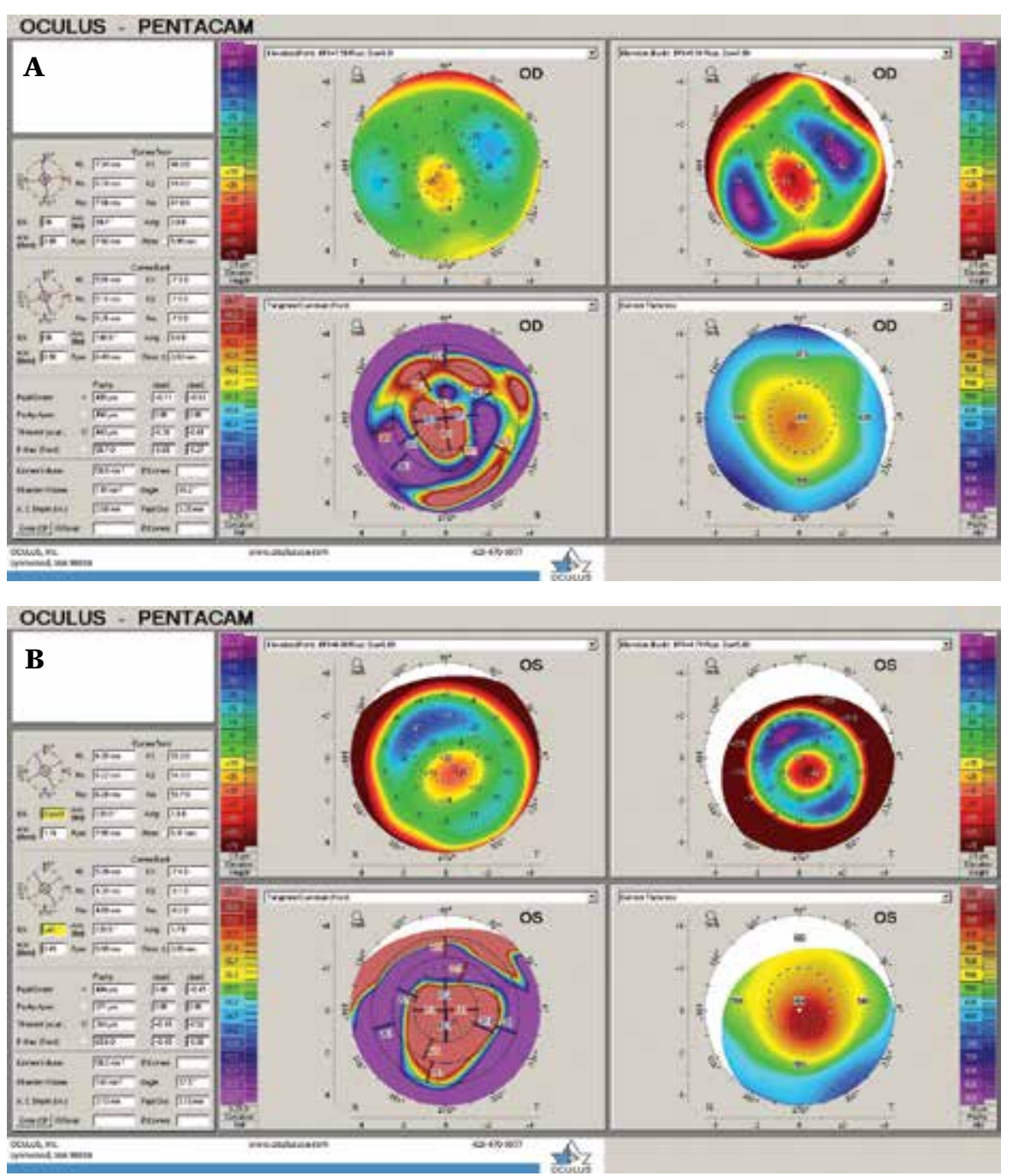

Figure 6. Most recent Pentacam topography maps for patient's (A) right and (B) left eyes taken in 2013. Both images show marked central steepening and apical thinning that is more advanced in the left eye. 
At the next follow-up visit, L.P. reported further improvement in his ocular comfort and that artificial tears were required less than once a day. The patient continues with the successful PBCL system and can wear the lenses for 16 hours a day. Corneal topographies were taken again; these are shown in Figure 6. The right eye had $3.8 \mathrm{D}$ (axis 99.7 ) of corneal astigmatism with corneal radii of flat $46.0 \mathrm{D}$ and steep $49.8 \mathrm{D}$ and thinnest pachymetry of $440 \mu \mathrm{m}$. The left eye had $1.1 \mathrm{D}$ (axis $120^{\circ}$ ) of corneal astigmatism with corneal radii of flat 53.2 D and steep 54.3 D and pachymetry of $344 \mu \mathrm{m}$ at the thinnest point. The topography readings in Figure 6 show obvious abnormalities with marked steepening and thinning of the corneas and decentered apices.

\section{DISCUSSION}

Development of corneal ectasia after refractive surgery is rare, but because the consequences for the patient can be so severe, it is important to prevent this complication. Risk factors for developing ectasia have been difficult to identify in the literature due to the rare incidence and contradicting information. Although it is assumed that removal of excessive amounts of corneal tissue results in a cornea that can be too weak to maintain its structural integrity, there are no consistent measurements for minimum preoperative pachymetry or residual stromal bed thickness (RSBT) that are agreed upon. While the minimum values considered most commonly are $500 \mu \mathrm{m}$ for preoperative pachymetry and $250 \mu \mathrm{m}$ for RSBT, there are still significant numbers of reports of patients with measurements greater than these values who develop ectasia, as well as measurements below these cut-offs who do not. ${ }^{11,13,29}$

Abnormalities in preoperative corneal topographies appear to be a more established risk factor. Most cases of iatrogenic keratoectasia have been in patients who showed topographic signs of keratoconus or pellucid marginal degeneration prior to the surgery. ${ }^{11,13,29}$ Other risk factors for developing ectasia after refractive surgery mentioned in the literature include younger age, higher refractive correction, steep preoperative corneal curvature, correction for against-the-rule or oblique astigmatism, number of enhancement procedures, eye rubbing,

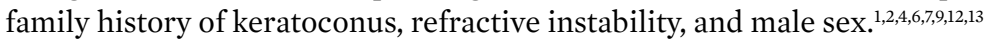

The patient presented here had preoperative pachymetry measurements of $521 \mu \mathrm{m}$ in the right eye and $522 \mu \mathrm{m}$ in the left eye and RSBT of $272 \mu \mathrm{m}$ in the right eye and $259 \mu \mathrm{m}$ in the left eye. The pachymetry values are symmetrical and both are greater than the 500 - $\mu \mathrm{m}$ cut-off mentioned. The RSBT measurements exceed the popular $250-\mu \mathrm{m}$ cut-off but are less than the more conservative $300 \mu \mathrm{m}$ minimum value. This supports the idea that one value for either pachymetry or RSTB cannot be applied to all cases. The preoperative corneal topographies for this patient show abnormalities, thus reinforcing this as an established risk factor. Because the standard of care regarding diagnosis of keratoconus and prevention of post-refractive ectasia has shown advancements over the past couple of decades, ${ }^{11}$ it may be more reasonable to consider that the topography abnormalities were undetected at the time. However, by today's standards, the preoperative maps are certainly suspicious, and this patient would be considered a non-candidate or possibly undergo further investigation before proceeding with the surgery.

In terms of other risk factors for post-LASIK ectasia mentioned in the literature, this patient was a male and young at the time of the procedure (aged 23 years). He also showed a very small amount of against-the-rule astigmatism in the left eye, which contrasts with the small amount of with-the-rule astigmatism in the right eye. This asymmetry between the eyes may be negligible, however, since the magnitude of astigmatism is so small. Notably, the eye that had againstthe-rule astigmatism (the left) showed more advanced ectasia. Other risk factors, such as high refractive correction, steep preoperative corneal curvatures, and number of enhancements, did not apply to this patient. There were no data regarding the stability of his refraction or history of eye rubbing from the time of the LASIK procedure.

Some literature identifies two distinct types of post-refractive ectasia. One entity is described as a central ectasia with little irregular astigmatism and good BCVA that occurs in normal eyes, and the other is described as a paracentral ectasia more similar to keratoconus, with irregular astigmatism and poor BCVA that more often occurs in eyes that have preoperative keratoconus, 
pellucid marginal degeneration, or forme fruste keratoconus..$^{8-10}$ The patient in this report aligns more with the paracentral ectasia more similar to keratoconus. Although there was no obvious irregular astigmatism seen on the corneal topography maps, there is a decentered apex and a reduced BCVA with spectacles in both eyes. Additionally, there were abnormalities in corneal topography prior to the procedure.

The ocular surface disease seen in this patient likely had multiple etiologies. Because the patient had suspicious preoperative corneal topographies, he might have developed keratoconus later in life, even if the LASIK procedure was not performed. If this were the case, he would likely have experienced ocular surface dryness and sensitivities commonly associated with noniatrogenic keratoconus. Furthermore, a poor lens cornea fitting relationship can exacerbate ocular irritation and dry eye signs and symptoms. Although ocular surface disease after LASIK is a common complication within the first few months, usually only a small proportion of patients develop chronic and severe dry eyes. ${ }^{1,2,24,25}$ The primary mechanism for post-LASIK dry eye appears to be a decreased feedback in the cornea-lacrimal gland functional unit as a result of the corneal denervation from flap creation and surface ablation. ${ }^{2,24-28}$ Persistent ocular surface disease may be better explained by abnormal nerve regeneration in the years following LASIK. ${ }^{2,24-28}$ Since this cannot be measured with clinical methods, it cannot be said for certain whether this was a cause of ocular surface disease in this patient.

Corneal ectasia and dry eye syndrome are challenging post-LASIK complications to treat in isolation, and their coexistence can compound problems, making management even more difficult. The PBCL system employed in this case provided an elegant solution for the difficult lens fit as a result of the irregularly shaped cornea and concomitant ocular surface disease. The soft lens improved centration of the overlying GP lens and reduced the area of bearing on the protruded corneal apices, as evidenced by the fluorescein patterns. The use of a minus power soft lens seemed to help reduce the area of central bearing because of the thinner central thickness profile of the lens design. Although a plus power soft lens might have further improved centration, adequate centration was achieved with the minus power. Thicker centre profile of a plus lens increased the area of central bearing and would have required a new GP lens order.

The improvement in lens fit and centration alone would be expected to aid in improving the condition of the ocular surface. The presence of the soft lens also acted as a bandage to protect the corneal surface from further desiccation and mechanical irritation caused by the GP lens. The overall effect of adding the soft lens was to promote a healthier environment for the corneal surface to heal.

The choice of using a daily disposable soft contact lens for the PBCL was twofold. First, use of a daily lens is ideal when lens deposits are a concern, because the lens has a shorter amount of time for deposits to accumulate. This was an important concern for our patient, because he developed lens deposit issues with his GP lenses, and ocular surface dryness can lead to increased surface deposits. Second, a daily lens is more convenient in terms of handling, since it reduces the number of lenses that require cleaning by half. The use of a silicone hydrogel material was preferred to increase the oxygen available to the eye to promote corneal healing and to avoid hypoxic complications. Adding a soft lens effectively decreases corneal oxygen delivery by increasing the amount of lens material between the atmosphere and the ocular surface. Corneal hypoxia can cause complications such as neovascularization, which may reduce the success of corneal surgery if required by the patient in the future. ${ }^{30}$ 


\section{CONCLUSION}

This case demonstrates successful use of a piggyback contact lens system in a patient with post-LASIK keratoectasia and concurrent ocular surface disease. The therapeutic soft contact lens in the piggyback system improved the fit of the RGP lens and provided protection of the ocular surface that promoted corneal healing and increased comfort. When using a piggyback contact lens system in a patient with ocular surface disease, it is important to consider the contact lens replacement schedule and cleaning regimen, as well as treatment with artificial tears. Successful use of this contact lens system can postpone or eliminate the need for surgical or pharmacological interventions.

\section{REFERENCES}

1. Sutton GL, Kim P. Laser in situ keratomileusis in 2010-a review. Clin Experiment Ophthalmol 2010;38:192-210.

2. Bromley JG, Albright TD, Kharod-Dholakia B, et al. Intraoperative and postoperative complications of laser in situ

keratomileusis. Expert Rev Ophthalmol 2012;7:25-31.

3. Binder PS, Lindstrom RL, Stulting RD, et al. Keratoconus and corneal ectasia after LASIK. J Cataract Refract Surg 2005;31:2035-8.

4. Randleman JB, Russell B, Ward MA, et al. Risk factors and prognosis for corneal ectasia after LASIK. Ophthalmology 2003;110:267-75.

5. Said A, Hamade IH, Tabbara KF. Late onset corneal ectasia after LASIK surgery. Saudi J Ophthalmol 2011;25:225-30.

6. Randleman JB, Woodward M, Lynn MJ, et al. Risk assessment for ectasia after corneal refractive surgery. Ophthalmology 2008;115:37-50. e4.

7. Brenner LF, Alió JL, Vega-Estrada A, et al. Clinical grading of post-LASIK ectasia related to visual limitation and predictive factors for vision loss. J Cataract Refract Surg 2012;38:1817-26.

8. Comaish IF, Lawless MA. Progressive post-LASIK keratectasia: biomechanical instability or chronic disease process? J Cataract Refract Surg 2002;28:2206-13.

9. Binder PS. Ectasia after laser in situ keratomileusis. J Cataract Refract Surg 2003;29:2419-29.

10. Faraj HG, Gatinel D, Chastang PJ, et al. Corneal ectasia after LASIK. J Cataract Refract Surg 2003;29:220.

11. Rabinowitz YS. Ectasia after laser in situ keratomileusis. Curr Opin Ophthalmol 2006;17:421-6.

12. Saad A, Gatinel D. Bilateral corneal ectasia after laser in situ keratomileusis in patient with isolated difference in central corneal thickness between eyes. J Cataract Refract Surg 2010;36:1033-5.

13. Binder PS. Analysis of ectasia after laser in situ keratomileusis: risk factors. J Cataract Refract Surg 2007;33:1530-8.

14. Marinho A, Pinto MC, Vaz F. Corneal ectasia after LASIK-how to manage. Semin Ophthalmol 2000;15:148-50.

15. Yeung KK, Olson MD, Weissman BA. Complexity of contact lens fitting after refractive surgery. Am J Ophthalmol 2002;133:607-12.

16. Choi H, Kim M, Lee J. Optimization of contact lens fitting in keratectasia patients after laser in situ keratomileusis. J Cataract Refract Surg 2004;30:1057-66.
17. Giasson CJ, Perreault N, Brazeau D. Oxygen tension beneath piggyback contact lenses and clinical outcomes of users. Eye Contact Lens 2001;27:144-50.

18. Javadi MA, Feizi S. Deep anterior lamellar keratoplasty using the big-bubble technique for keratectasia after laser in situ keratomileusis. J Cataract Refract Surg 2010;36:1156-60.

19. López-Alemany A, González-Méijome JM, Almeida JB, et al. Oxygen transmissibility of piggyback systems with conventional soft and silicone hydrogel contact lenses. Cornea 2006;25:214-19.

20. Sengor T, Kurna SA, Aki S, et al. High dk piggyback contact lens system for contact lens-intolerant keratoconus patients. Clin Ophthalmol 2011;5:331-5.

21. Romero-Jiménez M, Santodomingo-Rubido J, FloresRodríguez P, et al. Which soft contact lens power is better for piggyback fitting in keratoconus? Cont Lens Anterior Eye 2013;36:45-8.

22. O'Donnell C, Maldonado-Codina C. A hyper-Dk piggyback contact lens system for keratoconus. Eye Contact Lens 2004;30:44-8.

23. Yu EY, Leung A, Rao S, et al. Effect of laser in situ keratomileusis on tear stability. Ophthalmology 2000;107:2131-5.

24. Feng Y, Yu J, Wang D, et al. The effect of hinge location on corneal sensation and dry eye after LASIK: a systematic review and meta-analysis. Graefes Arch Clin Exp Ophthalmol 2013;251:357-66.

25. Shtein RM. Post-LASIK dry eye. Expert Rev Ophthalmol 2011;6:575-82.

26. Ambrosio R Jr, Tervo T, Wilson SE. LASIK-associated dry eye and neurotrophic epitheliopathy: pathophysiology and strategies for prevention and treatment. J Refract Surg 2008;24:396-407.

27. Rosenfeld SI. Evaluation and management of postLASIK dry eye syndrome. Int Ophthalmol Clin 2010;50:191-9.

28. Nettune GR, Pflugfelder SC. Post-LASIK tear dysfunction and dysesthesia. Ocul Surf 2010;8:135-45.

29. Salz JJ, Binder PS. Is there a "magic number" to reduce the risk of ectasia after laser in situ keratomileusis and photorefractive keratectomy? Am J Ophthalmol 2007;144:284-5

30. Bachmann B, Taylor RS, Cursiefen C. Corneal neovascularization as a risk factor for graft failure and rejection after keratoplasty. Ophthalmol 2010;117:1300-5. 